\title{
Comparison of inflammatory markers in non-dipper hypertension vs. dipper hypertension and in normotensive individuals: uric acid, C-reactive protein and red blood cell distribution width readings
}

\author{
Aydin Rodi Tosu ${ }^{1}$, Serafettin Demir ${ }^{2}$, Murat Selcuk ${ }^{1}$, Yuksel Kaya ${ }^{3}$, Aytac Akyol ${ }^{1}$, Mahmut Ozdemir $^{4}$, \\ Erhan Tenekecioglu \\ 1Department of Cardiology, Van Education and Research Hospital, Van, Turkey \\ 2Department of Cardiology, Adana Public Hospital, Adana, Turkey \\ ${ }^{3}$ Department of Cardiology, Kafkas University, Kars, Turkey \\ ${ }^{4}$ Department of Cardiology, Yuzuncu YII University, Van, Turkey \\ ${ }^{5}$ Department of Cardiology, Bursa Education and Research Hospital, Bursa, Turkey
}

Postep Kardiol Inter 2014; 10, 2 (36): 98-103

DOI: $10.5114 /$ pwki.2014.43514

\begin{abstract}
A bstract
Aim: In this study, we investigated the relationship of increased inflammatory parameters (C-reactive protein - CRP), oxidative stress markers (serum uric acid - SUA) and red blood cell distribution width (RDW) with non-dipper hypertension (NDHT).

Material and methods: Among the individuals who presented to the cardiology clinic, 40 patients (32.5\% male, 67.5\% female; mean age: $54.4 \pm 7.1$ ) who had hypertension and were diagnosed with NDHT through ambulatory blood pressure monitoring, 40 age- and sex-matched dipper hypertension (DHT) patients ( $25 \%$ male, $75 \%$ female, mean age: $54.2 \pm 7.0$ ), and 40 normotensive individuals $(42.5 \%$ male, $57.5 \%$ female, mean age: $51.9 \pm 9.0)$ were enrolled in the study. Peripheral venous blood samples were collected from all the patients in order to evaluate the hematological and biochemical parameters. All the assessed parameters were compared among the groups.

Results: The CRP, RDW and uric acid levels were observed to be significantly higher in the non-dipper hypertension group in comparison to the dipper hypertension patients and the normotensive population $(p<0.05)$. These parameters were also significantly higher in the dipper HT group compared to the normotensive population $(p<0.05)$.

Conclusions: We found in our study that increased CRP, uric acid and RDW levels, which are indicators of increased inflammation and oxidative stress, are significantly higher in the non-dipper HT patients in comparison to the dipper HT patients and control group.
\end{abstract}

Key words: non-dipper hypertension, dipper hypertension, uric acid, red blood cell distribution width, C-reactive protein.

\section{Introduction}

Blood pressure shows a circadian rhythm. In healthy individuals, blood pressure reaches a peak in the morning hours and slowly falls during the day to reach the lowest levels in the night. Both systolic and diastolic blood pressures decrease by more than $10 \%$ during the night compared to the daytime. Ambulatory blood pressure monitoring readings are more closely associated with a poor prognosis in comparison to blood pressure values measured in the clinical setting. The hypertensive patients were divided into dip- pers and non-dippers based on the ambulatory blood pressure monitoring results [1]. Hypertension $(H T)$, especially non-dipper $\mathrm{HT}$, which is defined as a reduction of less than $10 \%$ in the night time blood pressure compared to the daytime blood pressure, is a risk factor for cardiovascular disease and the associated adverse events [2-5]. Although the relationship between non-dipper $\mathrm{HT}$, increased target organ damage and the higher frequency of cardiovascular events is still unclear, increased inflammation and high platelet activity are thought to be responsible [6].

\section{Corresponding author:}

Serafettin Demir MD, Department of Cardiology, Adana Public Hospital, 01270, Adana, Turkey, phone/fax: +90 5336271991 ,

e-mail: demirkardiyoloji@hotmail.com

Received: 6.06.2013, accepted: 20.01.2014. 
There are findings showing that uric acid, which is the end product of purine catabolism in humans, is a component of the metabolic syndrome and a simple marker of insulin resistance. Increased serum uric acid (SUA) levels have been reported to be associated with increases in certain inflammatory markers and indicators of oxidative stress [7-9]. C-reactive protein (CRP) is a member of the pentaxin family, which is characterized by circular pentameric disc-shaped proteins. The synthesis of CRP occurs mainly in the liver through the influence of cytokines (of which the most important one is the interleukin-6 (IL-6)) secreted from the inflamed tissue [10]. An increased level of CRP is an important indicator of increased inflammation and endothelial dysfunction [11]. The red blood cell distribution width (RDW), which is mainly used for the differential diagnosis of anemia, is an automated measurement of the heterogeneity of the red blood cell size (anisocytosis). The bone marrow dysfunction caused by the increased systemic inflammation and the consequent increase in the ineffective erythropoiesis may lead to increased anisocytosis [12]. In various studies, baseline higher RDW, SUA and CRP values were shown to be associated with adverse events in cardiovascular diseases such as HT [13-15].

\section{Aim}

The aim of our study was to investigate the relationship between inflammatory markers and non-dipper HT, which is associated with increased end organ failure and cardiovascular adverse events.

\section{Material and methods}

For the purposes of our study, 80 patients with HT (40 non-dipper HT patients and 40 age- and sex-matched dipper HT patients), who had presented to the cardiology clinic of our hospital within the last year and were previously diagnosed with primary (essential) HT and followed up under treatment with the same group of anti-hypertensives (combination therapy with angiotensin-converting enzyme (ACE) inhibitors + thiazide) and 40 normotensive individuals (age- and sex-matched with the other groups) were enrolled. The classification of the dippers, non-dippers and the control group was based on ambulatory blood pressure monitoring. The patients' body mass indices (BMI) and smoking status were specified. Those with atrial fibrillation, history of myocardial infarction, left ventricular systolic dysfunction, left ventricular hypertrophy, renal and hepatic failure (serum creatinine $>1.5 \mathrm{mg} / \mathrm{dl}$ and aspartate aminotransferase-alanine aminotransferase (AST-ALT) > 2 times the normal values), hypothyroidism, hepatic dysfunction, hemolytic disease, concomitant inflammatory diseases such as autoimmune disorders, neoplastic disease, recent major surgery or any systemic disorders, diseases of the respiratory tract (chronic obstructive pulmonary disease, chronic bronchitis, pulmonary embolism), primary pulmonary $\mathrm{HT}$, isolated right heart failure, congenital heart disease and advanced valvular disease were excluded from the study.

\section{Blood pressure measurements}

Ambulatory blood pressure (BP) monitoring was performed using a non-invasive recording system (DiaSys Diagnostic Systems GmbH, Holzheim, Germany). The device was programmed to repeat the measurements every $15 \mathrm{~min}$ throughout the daytime ( $6 \mathrm{am}-8 \mathrm{pm})$ and every 30 min during the night ( $8 \mathrm{pm}-6 \mathrm{am})$ for $24 \mathrm{~h}$. Those patients in which the mean systolic and diastolic BP values measured during the night showed a $10 \%$ or greater decrease compared to the mean daytime values were classified into the DHT group, while those with decreases below $10 \%$ were classified into the non-DHT group.

\section{Transthoracic echocardiography}

The transthoracic echocardiography was carried out before the discharge by a system $\mathrm{V}$ (Vingmed; GE, Horten, Norway) device and using a $2.5 \mathrm{MHz}$ phased-array transducer. Recordings were performed while the patients were in the left lateral decubitus position. The LV ejection fraction was measured using the modified Simpson's rule according to the latest guidelines [16].

\section{Statistical analysis}

Continuous variables are expressed as mean \pm SD. Categorical variables are expressed in percentages. In order to compare the parametric continuous variables, Student's t-test or analysis of variance was used, while the nonparametric continuous variables were compared using the Mann-Whitney $U$ or Kruskal-Wallis tests. The categorical variables were compared through the $\chi^{2}$ test. Two-tailed $p$ values $<0.05$ were considered to indicate statistical significance. Statistical analyses were performed using SPSS version 20.0 for Windows.

\section{Definitions}

The BMI was calculated by dividing the weight in $\mathrm{ki}$ lograms by the height in square meters $\left(\mathrm{kg} / \mathrm{m}^{2}\right)$. In line with the World Health Organization criteria, anemia at presentation was defined as a baseline haemoglobin $(\mathrm{Hgb})$ concentration below $13 \mathrm{mg} / \mathrm{dl}$ in males and below $12 \mathrm{mg} / \mathrm{dl}$ in females. The diagnosis of diabetes mellitus (DM) was based on previous history of diabetes treated with or without medical therapy. Stable angina was defined as discomfort in the chest, jaw, shoulder, back, or arms, typically elicited by exertion or emotional stress and relieved by rest or nitroglycerin. Current smokers were defined as those who had smoked for some period during the past year. Those patients dependent on chronic dialysis were considered as having end stage renal disease (ESRD). The approval of the local ethics committee was obtained for this study. 


\section{Results}

Among the 120 patients (mean age $53.5 \pm 7.7 ; 33.3 \%$ male), RDW (mean value: $13.9 \pm 1.2 \%$ ), CRP (mean value: $1.4 \pm 0.3$ ) and SUA (mean value: $5.2 \pm 0.8$ ) studied. The baseline characteristics of the normotensive individuals and the patients with dipper and non-dipper HT are summarized in Table I. No statistically significant difference was observed among the three groups in terms of smoking status, fasting blood glucose, and HDL and LDL values $(p>0.05)$. While the BMI value in the control group was significantly lower than the dipper HT and non-dipper HT groups $(p<0.05)$, the BMI value in the non-dipper HT group was not significantly different from the dipper HT group $(p>0.05)$. The triglyceride value in the control group was significantly higher than the dipper HT group $(p<0.05)$; however, the difference in the triglyceride value of the non-dipper HT group from the dipper HT and control groups was statistically insignificant $(p>0.05)$.

The RDW readings in the non-dipper HT group were significantly higher than the dipper HT and control groups $(p<0.05)$. Also, the RDW values in the dipper HT group were significantly higher than the control group $(p<0.05)$. While the CRP and SUA values in the non-dipper HT group were significantly higher than the dipper HT and control groups $(p<0.05)$, the differences in the CRP and SUA values between the dipper HT and control groups were insignificant $(p>0.05)$ (Table II, Figures 1-3).

\section{Discussion}

In the study, we found that the CRP, SUA and RDW levels were significantly higher in the non-dipper HT patients compared to dipper HT patients and normotensive individuals. Also, we observed that the RDW levels were higher in the dipper HT patients compared to normotensive individuals.

Cardiovascular parameters such as BP heart rate, and coronary tonus change with the circadian rhythm during the day [17]. According to the ambulatory blood pressure monitoring (ABPM) data obtained from normotensive individuals, the BP reaches the highest values in the morning hours and gradually drops during the day to reach the lowest values throughout the night [18]. This circadian rhythm of the blood pressure has led to a new classification based on ambulatory blood pressure monitoring: the type of BP in which the BP values measured during the night are $\geq 10 \%$ lower than the blood pressure value readings during the day is defined as dipper HT (DHT), whereas the type of HT where this difference is less than $10 \%$ is described as non-dipper HT (NDHT) [19]. It has also been observed that the circulating free norepinephrine levels and the peripheral vascular resistance are increased in patients with NDHT above the age of 55 [17]. Therefore, an inadequate drop in BP occurs in these patients during the night. Higher rates of cerebrovascular disease, left ventricular mass, and increase in cardiovascular mortality and morbidity have been re-

Table I. Demographic characteristics and laboratory findings of the groups

\begin{tabular}{|c|c|c|c|c|c|}
\hline \multicolumn{2}{|c|}{ Parameter } & \multirow{2}{*}{$\begin{array}{c}\begin{array}{c}\text { Control group } \\
\text { Mean } \pm \text { SD/n (\%) }\end{array} \\
51.3 \pm 9.0\end{array}$} & \multirow{2}{*}{$\begin{array}{c}\begin{array}{c}\text { Dipper HT } \\
\text { Mean } \pm \text { SD/n (\%) }\end{array} \\
54.2 \pm 7.0\end{array}$} & \multirow{2}{*}{$\begin{array}{c}\begin{array}{c}\text { Non-dipper HT } \\
\text { Mean } \pm \text { SD/n (\%) }\end{array} \\
54.2 \pm 7.1\end{array}$} & \multirow{2}{*}{$\frac{\text { Value of } p}{0.290}$} \\
\hline Age [years] & & & & & \\
\hline \multirow[t]{2}{*}{ Sex } & Male & $17(42.5)$ & $10(25.0)$ & $13(32.5)$ & \multirow[t]{2}{*}{0.250} \\
\hline & Female & $23(57.5)$ & $30(75.0)$ & $27(67.5)$ & \\
\hline \multirow[t]{2}{*}{ Smoking } & Yes & $16(40.0)$ & $11(27.5)$ & $14(35.0)$ & \multirow[t]{2}{*}{0.495} \\
\hline & No & $24(60.0)$ & $29(72.5)$ & $26(65.0)$ & \\
\hline \multicolumn{2}{|l|}{$\mathrm{BMI}\left[\mathrm{kg} / \mathrm{m}^{2}\right]$} & $25.3 \pm 2.1$ & $27.6 \pm 2.5$ & $27.0 \pm 2.2$ & $<0.001$ \\
\hline \multicolumn{2}{|l|}{ Glucose } & $97.5 \pm 23.0$ & $103.2 \pm 17.1$ & $104.7 \pm 19.0$ & 0.102 \\
\hline \multicolumn{2}{|l|}{$\mathrm{HDL}$} & $41.3 \pm 3.1$ & $41.5 \pm 4.5$ & $40.2 \pm 3.5$ & 0.253 \\
\hline \multicolumn{2}{|l|}{ LDL } & $106.0 \pm 29.6$ & $119.2 \pm 29.7$ & $119.5 \pm 29.6$ & 0.072 \\
\hline \multicolumn{2}{|l|}{ Triglyceride } & $193.1 \pm 15.4$ & $178.9 \pm 24.0$ & $188.5 \pm 22.8$ & 0.011 \\
\hline
\end{tabular}

$B M I$ - body mass index, HDL - high-density lipoprotein cholesterol, $L D L$ - low-density lipoprotein cholesterol

Table II. Blood RDW, CRP and uric acid values of the groups

\begin{tabular}{lccc} 
Parameter & $\begin{array}{c}\text { Control group } \\
\text { Mean } \pm \text { SD }\end{array}$ & $\begin{array}{c}\text { Dipper HT } \\
\text { Mean } \pm \text { SD }\end{array}$ & $\begin{array}{c}\text { Non-dipper HT } \\
\text { Mean } \pm \text { SD }\end{array}$ \\
\hline RDW [\%] & $11.8 \pm 2.1$ & $14.3 \pm 0.8$ & $15.6 \pm 0.9$ \\
\hline C-reactive protein & $1.0 \pm 0.3$ & $1.4 \pm 2.2$ & $1.8 \pm 0.5$ \\
\hline Uric acid & $4.5 \pm 1.0$ & $4.7 \pm 0.7$ & $6.6 \pm 0.9$
\end{tabular}




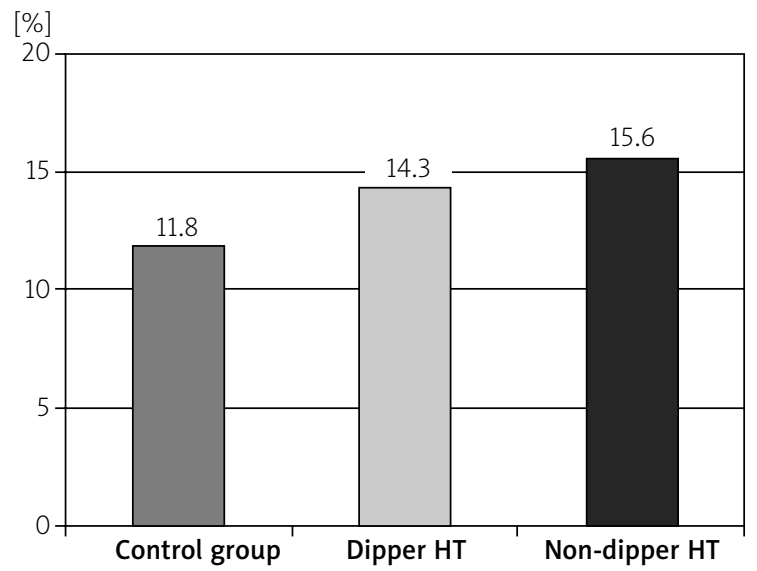

Figure 1. The RDW levels of the groups

ported in patients with non-dipper HT. Also, higher BP readings during the night were observed to be associated with left ventricular hypertrophy (LVH) [20-22].

In non-dipper hypertensive patients, higher blood pressure levels during the night lead to greater endothelial damage, while greater endothelial damage leads to higher levels of BP during the night [23]. The increase in the blood pressure triggers the proinflammatory process within the endothelium of the blood vessels and the consequent endothelial inflammation leads to higher CRP levels. In the study by Gawin et al. including 15215 female patients, a linear relationship was found between the CRP levels and the BP levels [24]. The results of this study showed that the inflammation leads to higher blood pressure, while higher blood pressure worsens the inflammation. The increase caused by CRP in the angiotensin-II and endothelin-I levels besides the decrease in the nitric oxide (NO) levels is an example of these conditions. In the study by Bautista et al. the CRP levels in hypertensive patients were evaluated and CRP was identified as a potential independent risk factor in the development of HT [25]. In another study conducted on a Korean population, it was observed as an independent predictor of HT. In our study, the CRP levels in the non-dipper and dipper HT groups were found to be higher than the normal group. Also, the comparison of the CRP readings between the non-dipper and dipper HT groups revealed higher values in the non-dipper group.

Hyperuricemia is an indirect indicator of increased oxidative stress. During the synthesis of uric acid, hydrogen peroxide - a very reactive type of oxygen - is generated. Hyperuricemia is associated with HT, vascular disease, renal disease and cardiovascular events. The results of a study reported that increased serum uric acid levels were associated with a greater frequency of HT [26]. In another study, higher serum uric acid levels were found to be an independent risk factor for chronic renal failure, which is in turn an independent risk factor for HT and non-dipper HT [27]. Kanbay et al. observed an increase in

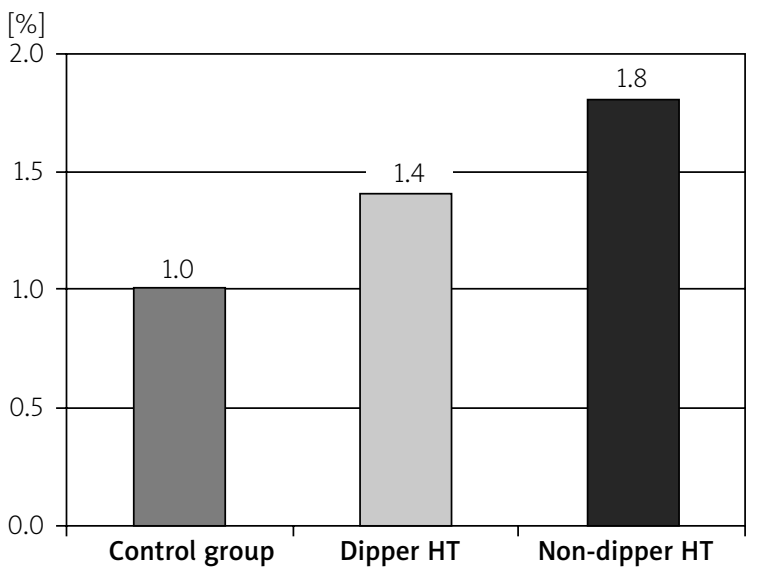

Figure 2. The CRP levels of the groups

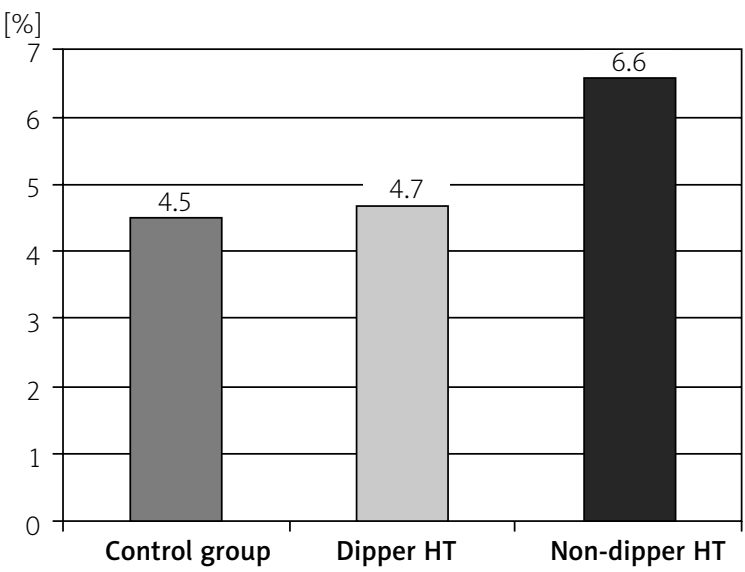

Figure 3. The uric acid levels of the groups

the glomerular filtration rates and decrease in the systolic and diastolic blood pressures of patients with normal renal function treated with allopurinol against hyperuricemia [27]. The decrease in the renal blood flow is known to stimulate the re-absorption of uric acid [28]. Hypertension also causes microvascular disease and this leads to local tissue ischemia. Ischemia increases the synthesis of uric acid besides the release of lactate that hinders the production of uric acid in the proximal tubule [29]. In our study, the uric acid levels which were found to be higher in comparison to the control group may be a result of this mechanism.

While the RDW is a marker that shows the erythrocyte distribution volume, it also has come to be used in recent years as a marker that shows the increase in the morbidity and mortality of cardiovascular diseases. In a study by Çetin et al., a relationship between higher RDW readings and widespread vascular involvement in the coronary angiographies was found in patients with stable angina [30]. In the study by Tanindi et al., prehypertensive and hypertensive patients were compared 
with the control group and the RDW levels were found to be higher independently from the other parameters. Also in the same study, the RDW levels were demonstrated to be closely associated with the increases in the systolic and diastolic blood pressures [31]. Jithesh et al. evaluated the hs-CRP and RDW levels in hypertensive patients and observed that both parameters were higher in the hypertensive patient group compared to the control group [32]. Also in our study, the RDW levels in the non-dipper HT group were found to be significantly higher than the dipper HT and control groups. In addition, the RDW levels in the dipper HT group were significantly higher than the control group. The pathophysiological mechanism of the relationship between higher RDW values and the development of HT and non-dipper HT is yet to be clarified. However, the most plausible hypothesis is that higher RDW levels are associated with increased inflammation and oxidative stress. Indeed, we observed a correlation between the RDW levels and the CRP and SUA readings. In non-dipper hypertensive patients, the increased inflammation and oxidative stress might be causing the immature erythrocytes in the bone marrow to enter the circulation, thus leading to anisocytosis (and increased RDW levels).

\section{Conclusions}

We observed in our study that the CRP, uric acid and RDW levels were statistically significantly higher in non-dipper HT in comparison to the dipper HT and control groups. Higher levels of end-organ failure are known to be associated with non-dipper HT compared to dipper $\mathrm{HT}$. Therefore, these increased markers in non-dipper HT may help explain the increased end-organ failure as well as the cardiovascular morbidity and mortality.

\section{References}

1. Fujii T, Uzu T, Nishimura M, et al. Circadian rhythm of natriuresis is disturbed in nondipper type of essential hypertension. Am J Kidney Dis 1999; 33: 29-35.

2. Shimada K, Kario K. Altered circadian rhythm of blood pressure and cerebrovascular damage. Blood Press Monit 1997; 2: 333-8.

3. Timio M, Venanzi S, Lolli S, et al. "Non-dipper" hypertensive patients and progressive renal insufficiency: a 3-year longitudinal study. Clin Nefrol 1995; 43: 382-7.

4. Verdecchia P, Porcellati C, Schillaci G, et al. Ambulatory blood pressure: an independent predictor of prognosis in essential hypertension. Hypertension 1994; 24: 793-801.

5. Ohkubo T, Hozawa A, Yamaguchi J, et al. Prognostic significance of the nocturnal decline in blood pressure in individuals with and without high 24-h blood pressure: the Ohasama study. J Hypertens 2002; 20: 2183-9.

6. Kaya MG, Yarlioglues M, Gunebakmaz O, et al. Platelet activation and inflammatory response in patients with nondipper hypertension. Atherosclerosis 2010; 209: 278-82.

7. Tomita M, Mizuno S, Yamanaka $\mathrm{H}$, et al. Does hyper-uricemia affect mortality? A prospective cohort study of Japanese male workers. J Epidemiol 2000; 10: 403-9.
8. Liese AD, Hense HW, Lowel $\mathrm{H}$, et al. Association of serum uric acid with all-cause and cardiovascular disease mortality and incident myocardial infarction in the MONICA Augsburg cohort. World Health Organization Monitoring Trends and Determinants in Cardiovascular Diseases. Epidemiology 1999; 10: 391-7.

9. Franse LV, Pahor M, Di Bari M, et al. Serum uric acid, diuretic treatment and risk of cardiovascular events in the Systolic Hypertension in the Elderly Program (SHEP). J Hypertens 2000; 18: 1149-54.

10. Vigushin DM, Pepys MB, Hawkins PN. Metabolic and scintigraphic studies of radioiodinated human C-reactive protein in health and disease. J Clin Invest 1993; 91: 135.

11. Targher G, Bertolini L, Zoppini G, et al. Increased plasma markers of inflammation and endothelial dysfunction and their association with microvascular complications in type 1 diabetic patients without clinically manifest macroangiopathy. Diabet Med 2005; 22: 999-1004.

12. Ozcan F, Turak O, Durak A, et al. Red cell distribution width and inflammation in patients with non-dipper hypertension. Blood Press 2013; 22: 80-5.

13. Uyarel H, Isik T, Ayhan E, Ergelen M. Red cell distrubition width (RDW): a novel risk factor for cardiovascular disease. Int J Cardiol 2011; 154: 351-2.

14. Feig DI, Kang DH, Johnson RJ. Uric acid and cardiovascular risk. N Engl J Med 2008; 359: 1811-21.

15. Pfützner A, Forst T. High-sensitivity C-reactive protein as cardiovascular risk marker in patients with diabetes mellitus. Diabetes Technol Ther 2006; 8: 28-36.

16. Lang RM, Bierig M, Devereux RB, et al. Recommendations for chamber quantification: a report from the American Society of Echocardiography's guidelines and standards committee and the chamber quantification writing group. J Am Soc Echocardiogr 2005; 18: 1440-63.

17. Millar-Craig MW, Bishop CN, Raftery EB. Circadian variation of blood-pressure. Lancet 1978; 1: 795-7.

18. Seo WS, Oh HS. The circadian rhythms of blood pressure and heart rate in the hypertensive subjects: dippers and non-dippers. Yonsei Med J 2002; 43: 320-8.

19. Fujii T, Uzu T, Nishimura M, et al. Circadian rhythm of natriuresis is disturbed in nondipper type of essential hypertension. Am J Kidney Dis 1999; 33: 29-35.

20. Pierdomenico SD, Costantini F, Bucci A, et al. Blunted nocturnal fall in blood pressure and oxidative stress in men and women with essential hypertension. Am J Hypertens 1999; 12: 356-63.

21. Ohkubo T, Imai Y, Tsuji I, et al. Relation between nocturnal decline in blood pressure and mortality. The Ohasama Study. Am J Hypertens 1997; 10: 1201-7.

22. Verdecchia P, Schillaci G, Porcellati C. Dippers versus non-dippers. J Hypertens Suppl 1991; 9: S42-4.

23. Higashi Y, Nakagawa K, Kimura M, et al. Circadian variation of blood pressure and endothelial function in patients with essential hypertensiona comparison of dippers and non-dippers. J Am Coll Cardiol 2002; 40: 2039-43.

24. Blake GJ, Rifai N, Buring JE, Ridker PM. Blood pressure, C-reactive protein, and risk of future cardiovascular events. Circulation 2003; 108: 2993-9.

25. Bautista LE, López-Jaramillo P, Vera LM, et al. Is C-reactive protein an independent risk factor for essential hypertension? J Hypertens 2001; 19: 857-61.

26. Feig DI, Nakagawa T, Karumanchi SA, et al. Hypothesis: uric acid, nephron number, and the pathogenesis of essential hypertension. Kidney Int 2004; 66: 281-7. 
27. Kanbay M, Ozkara A, Selcoki Y, et al. Effect of treatment of hyperuricemia with allopurinol on blood pressure, creatinine clearence, and proteinuria in patients with normal renal functions. Int Urol Nephrol 2007; 39: 1227-33.

28. Messerli FH, Frohlich ED, Dreslinski GR, et al. Serum uric acid in essential hypertension: an indicator of renal vascular involvement. Ann Intern Med 1980; 93: 817-21.

29. Friedl HP, Till GO, Trentz O, Ward PA. Role of oxygen radicals in tourniquet-related ischemia-reperfusion injury of human patients. Klin Wochenschr 1991; 69: 1109-12.

30. Çetin M, Kocaman SA, Bostan M, et al. Red blood cell distribution width (RDW) and its association with coronary atherosclerotic burden in patients with stable angina pectoris. Eur J Gen Med 2012; 9: 7-13.

31. Tanindi A, Topal FE, Topal F, Celik B. Red cell distribution width in patients with prehypertension and hypertension. Blood Pressure 2012; 21: 177-81.

32. Jithesh TK, Mathew R, Jayapal V, Vijayakumar T. Red cell distribution width and high sensitivity C-reactive protein as risk markers in hypertension. Int J Med Sci Public Health 2012; 1: $138-42$. 